\title{
Erratum to: Lysobacter fragariae sp. nov. and Lysobacter rhizosphaerae sp. nov. isolated from rhizosphere of strawberry plant
}

\author{
Hina Singh • Juan Du - Hien T. T. Ngo $\cdot$ KyungHwa Won • \\ Jung-Eun Yang $\cdot$ Ki-Young Kim • Tae-Hoo Yi
}

Published online: 24 April 2015

(C) Springer International Publishing Switzerland 2015

\section{Erratum to: Antonie van Leeuwenhoek DOI 10.1007/s10482-015-0439-x}

In the original published version of the article, the descriptions of Table 1 and Table 2 have been changed without changing the whole table. The corrected version of the tables provided in the erratum.

The online version of the original article can be found under doi:10.1007/s10482-015-0439-x.

H. Singh · J. Du · H. T. T. Ngo · K. Won ·

J.-E. Yang · T.-H. Yi ( $\bowtie)$

Department of Oriental Medicine Biotechnology, College

of Life Science, Kyung Hee University, Global Campus,

1732 Deokyoungdaero, Giheung-gu, Yongin-si,

Gyeonggi-do 446-701, Republic of Korea

e-mail: drhoo@khu.ac.kr

\section{K.-Y. Kim}

Department of Genetic Engineering, College of Life

Science, Kyung Hee University, Global Campus, 1732

Deokyoungdaero, Giheung-gu, Yongin-si,

Gyeonggi-do 446-701, Republic of Korea 
Table 1 Cellular fatty acid composition of strains THG-DN8.7T, THGDN8.3 $\mathrm{T}$ and the type strains of closely related species

\begin{tabular}{|c|c|c|c|c|c|c|c|}
\hline Fatty acid & 1 & 2 & 3 & 4 & 5 & 6 & 7 \\
\hline \multicolumn{8}{|l|}{ Saturated } \\
\hline $\mathrm{C}_{16: 0}$ & 8.5 & 5.9 & 6.0 & 9.1 & 11.7 & 6.9 & 9.8 \\
\hline \multicolumn{8}{|l|}{ Unsaturated } \\
\hline $\mathrm{C}_{16: 1} \omega 7 c$ alcohol & 8.0 & 4.7 & 7.7 & 7.9 & $\operatorname{Tr}$ & 3.6 & $\operatorname{Tr}$ \\
\hline Iso- $\mathrm{C}_{17: 1} \omega 9 c$ & 16.0 & 12.0 & 11.0 & 5.7 & 5.1 & 16.5 & 8.3 \\
\hline \multicolumn{8}{|l|}{ Branched-chain } \\
\hline Iso- $\mathrm{C}_{11: 0}$ & 3.9 & 3.4 & 4.7 & 3.2 & 1.3 & 5.2 & 3.3 \\
\hline Iso- $\mathrm{C}_{11: 0} 3 \mathrm{OH}$ & 5.6 & 4.6 & 7.7 & 5.2 & 1.8 & 6.5 & 6.6 \\
\hline Iso- $\mathrm{C}_{14: 0}$ & 1.6 & 5.8 & 3.2 & 4.1 & 1.4 & 1.7 & $\operatorname{Tr}$ \\
\hline Iso- $\mathrm{C}_{15: 0}$ & 12.4 & 11.4 & 18.6 & 12.9 & 6.4 & 17.7 & 20.5 \\
\hline Anteiso- $\mathrm{C}_{15: 0}$ & $\operatorname{Tr}$ & 2.5 & 5.9 & 4.2 & 5.9 & 2.9 & 3.5 \\
\hline Iso- $\mathrm{C}_{16: 0}$ & 22.4 & 31.2 & 16.4 & 23.5 & 6.9 & 17.3 & 14.0 \\
\hline Iso- $\mathrm{C}_{17: 0}$ & 5.7 & 1.7 & 3.8 & 1.8 & 2.1 & 9.9 & 1.8 \\
\hline
\end{tabular}

Strains 1, THG-DN8.7 ${ }^{\mathrm{T}} ; 2$, THG-DN8.3 ${ }^{\mathrm{T}} ; 3$, L. terrae KACC $17646^{\mathrm{T}} ; 4$, L. yangpyeongensis KACC $114087^{\mathrm{T}} ; 5$, L. niabensis $\mathrm{KACC}$ $11587^{\mathrm{T}}$; 6, L. oryzae KCTC $22249^{\mathrm{T}}$; 7, L. enzymogenes ATCC $29487^{\mathrm{T}}$. All the data were obtained in this study, cells were cultured on R2A agar for 2 days at $28{ }^{\circ} \mathrm{C}$

$\operatorname{Tr}$ traces $(<1.0 \%)$ 
Table 2 The differential biochemical and physiological characteristics of strains THG-DN8.7T and THGDN8.3T and closely related type strains of species of genus Lysobacter
Strains 1 , THG-DN8.7 $7^{\mathrm{T}} ; 2$, THG-DN8. $3^{\mathrm{T}} ; 3$, L. terrae KACC $17646^{\mathrm{T}} ; 4, \mathrm{~L}$. yangpyeongensis $\mathrm{KACC}$ $11407^{\mathrm{T}}$; 5, L. niabensis KACC $11587^{\mathrm{T}}$; 6, L. oryzae КСТC $22249^{\mathrm{T}} ; 7, \quad L$. enzymogenes ATCC $29487^{\mathrm{T}}$ (type strain of the genus). All the data were obtained in this study

$(+)$ Positive, $(W)$ weakly positive, (-) negative

\begin{tabular}{|c|c|c|c|c|c|c|c|}
\hline Characteristic & 1 & 2 & 3 & 4 & 5 & 6 & 7 \\
\hline Oxidase & + & - & + & + & + & + & + \\
\hline Motility & - & - & - & + & + & - & + \\
\hline Nitrate reduction & - & - & - & + & - & - & - \\
\hline \multicolumn{8}{|l|}{ Hydrolysis of } \\
\hline Starch & - & + & - & + & - & + & + \\
\hline Casein & + & + & + & + & - & + & + \\
\hline $\mathrm{CMC}$ & + & - & + & + & + & + & + \\
\hline Tween 20 & - & + & + & - & + & - & - \\
\hline Tween 80 & - & - & + & + & + & + & + \\
\hline Gelatin & + & + & $\mathrm{W}$ & + & $\mathrm{W}$ & W & + \\
\hline L-tyrosine & + & + & + & + & + & + & + \\
\hline DNA & - & + & - & + & + & - & - \\
\hline Esculin & - & - & + & - & + & - & + \\
\hline \multicolumn{8}{|l|}{ Assimilation of } \\
\hline$\beta$-Galactosidase & - & - & + & W & $\mathrm{W}$ & + & + \\
\hline$N$-acetyl-glucosamine & - & - & - & - & - & - & + \\
\hline D-Maltose & - & - & - & - & - & - & + \\
\hline Trisodium citrate & - & - & - & - & - & + & - \\
\hline Malic acid & - & - & - & - & - & - & + \\
\hline Capric acid & - & - & + & - & - & - & + \\
\hline \multicolumn{8}{|l|}{ Enzyme activity } \\
\hline Lipase (C14) & - & - & $\mathrm{W}$ & + & + & $\mathrm{W}$ & + \\
\hline Valine arylamidase & W & + & + & + & + & + & + \\
\hline Cysteine arylamidase & - & + & + & + & + & + & + \\
\hline Trypsin & - & + & + & + & $\mathrm{W}$ & + & + \\
\hline$\alpha$-Chymotrypsin & - & $\mathrm{W}$ & + & + & $\mathrm{W}$ & + & + \\
\hline Naphtol-AS-BI-phosphohydrolase & W & + & + & + & + & + & + \\
\hline$\alpha$-Glucosidase & - & - & $\mathrm{W}$ & + & + & + & + \\
\hline$\beta$-Glucosidase & - & - & + & + & + & + & + \\
\hline$N$-acetyl- $\beta$-glucosaminidase & - & + & + & + & - & - & + \\
\hline
\end{tabular}

